\title{
Analysis of the Atrial Repolarisation Phase of the Electrocardiogram in Health and in Atrial Fibrillation
}

\author{
P Langley $^{1,2}$, A Murray ${ }^{1,2}$ \\ ${ }^{1}$ University of Newcastle, Newcastle upon Tyne, UK \\ ${ }^{2}$ Freeman Hospital, Newcastle upon Tyne, UK
}

\begin{abstract}
Little is known about the atrial repolarisation phase of the ECG and it may provide a useful marker for those susceptible to atrial arrhythmia.

The amplitude of the atrial repolarisation phase of the $E C G$ and $P$ wave was measured in five patients with healthy hearts and in five patients with paroxysmal atrial fibrillation (PAF) who were in sinus rhythm at the time of the study. Atrial repolarisation amplitude was (mean (sd)) 15 (9) $\mu V$ (healthy), 17 (8) $\mu V$ (PAF) and $P$ wave amplitude was 100 (33) $\mu \mathrm{V}$ (healthy) and 102 (32) $\mu \mathrm{V}$ (PAF) with no significant differences between groups. There was a significant correlation between absolute values of $P$ wave amplitude and repolarisation amplitude (Pearson correlation 0.43, $p<0.05$ ). There was a negative association between depolarisation and repolarisation in the atria, opposite to that known for the ventricles.

The observable atrial repolaristion in PAF patients was no different to that in subjects with healthy hearts.
\end{abstract}

\section{Introduction}

Abnormal ventricular repolarisation in the ECG is a key marker for ventricular arrhythmia. For example, long QT syndrome is associated with increased risk of sudden death from torsade de pointes. Abnormal ventricular repolarisation on the ECG provides important diagnostic information, for example, indicating cardiac ischemia or myocardial infarction. Atrial repolarisation has not been studies so widely and very little is known about the atrial repolarisation phase of the ECG. This is not surprising given that much of the atrial repolarisation phase of the ECG is unobservable because it is obscured by the large ventricular activity. Additionally, the observable portion of the body surface atrial repolarisation signal is small and signal averaging is necessary to obtain a reasonable signal to noise ratio [1]. Invasive studies indicate that atrial repolarisation is abnormal in patients with paroxysmal atrial fibrillation [2] and our study investigated if the observable atrial repolarisation phase of the ECG was different in patients with atrial fibrillation compared to healthy subjects.

\section{Methods}

12-lead ECGs of 5 patients with paroxysmal atrial fibrillation and 5 healthy subjects without known heart disease were recorded for a duration of $600 \mathrm{~s}$ each. ECGs were sampled at $500 \mathrm{~Hz}$ with an amplitude resolution of less than $5 \mu \mathrm{V}$.

Figure 1 illustrates the processing steps used in the measurement of atrial repolarisation and $\mathrm{P}$ wave amplitude.

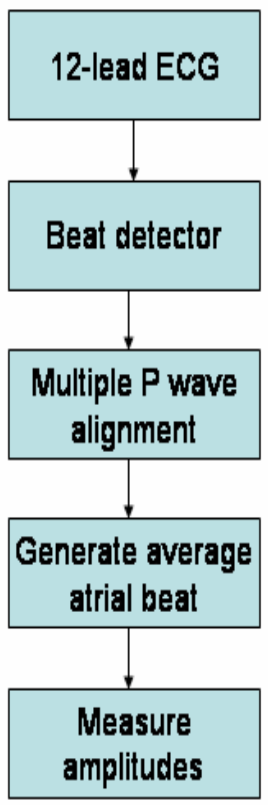

Figure 1. Processing steps for generating average 
atrial beats and amplitude measurements.

Atrial repolarisation amplitude is small and beat averaging is necessary to obtain signals with good signal to noise ratio. A QRS detector was used to identify individual cardiac beats throughout the entire recording.

Knowing the locations of QRS in each beat, the next step was to define a window containing the atrial beat $(\mathrm{P}$ wave and visible atrial repolarisation section). For each ECG this window initially covered an interval of $250 \mathrm{~ms}$ and started $300 \mathrm{~ms}$ before the $\mathrm{R}$ wave peak in each beat, but was subsequently manually adjusted to ensure the window contained no ventricular activity.

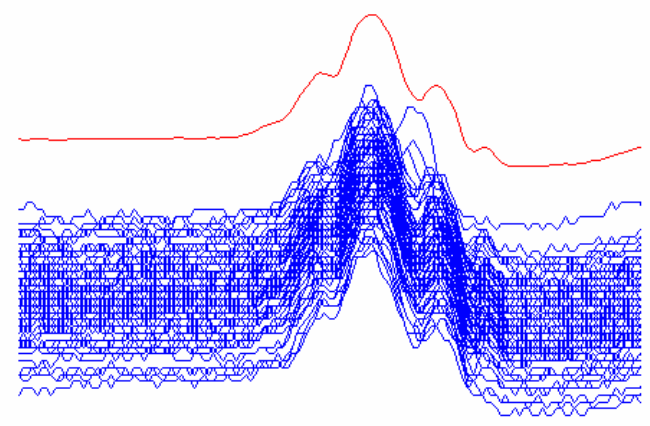

Figure 2. Collection of aligned $\mathrm{P}$ waves from a single recorded lead with averaged beat above.

The multiple atrial beats were time aligned using correlation, so that the maximum correlation between beats was obtained and only beats with correlations over a threshold were used to generate the average atrial beat in each lead. Figure 2 shows an example of the collection of atrial beats from a single lead of a subject along with the average beat.

Lead 'DC' offset was removed from the average beat by subtracting the mean amplitude of the 'isoelectric' interval before the onset of $P$ wave.

Figure 3 shows examples of average atrial beats in all leads from a healthy subject and PAF patient.

Amplitudes of $\mathrm{P}$ wave and atrial repolarisation phase of the atrial beat were measured manually. Amplitude was measured relative to zero isoelectric level.

\section{Results}

Figure 4 shows the atrial repolarisation and $\mathrm{P}$ wave amplitude measurements for all subjects and for all leads. Additionally, the ratio of repolarisation amplitude to $\mathrm{P}$ wave amplitude is shown.

Pooling the data from all the leads there were no significant differences (Student t-test, $\mathrm{p}>0.05$ ) between groups for absolute values of both atrial repolarisation amplitude (mean (sd) healthy 15 (9) $\mu \mathrm{V}$, PAF $17(8) \mu \mathrm{V}$ ) and $\mathrm{P}$ wave amplitude (mean (sd) healthy $100(33) \mu \mathrm{V}$, PAF $102(32) \mu \mathrm{V})$.

Combining data from both groups there was a significant correlation between absolute values of $\mathrm{P}$ wave amplitude and repolarisation amplitude (Pearson correlation 0.43, $\mathrm{p}<0.05$ ).

Comparing individual leads, mean repolarisation amplitude was negative, the opposite polarity to the $\mathrm{P}$ wave, in leads I, II, aVL, aVF, V2, V3, V4, V5, V6. In aVR repolarisation was positive and the $\mathrm{P}$ wave negative. Thus, there is a negative association between depolarisation and repolarisation in the atria, opposite to that known for the ventricles.

\section{Discussion and conclusions}

Our pilot study in this small group of patients suggests that there is no difference in the amplitude of the observable section of the atrial repolarisation phase of the ECG in patients with PAF compared with healthy subjects. Of course we have only studied a small part of the atrial repolarisation phase and it could be that abnormalities are manifest only in the unobservable later stages of repolarisation. There are considerable difficulties measuring the atrial repolarisation phase of the ECG because the amplitudes are small and much of the phase is obscured by ventricular depolarisation. Atrial repolarisation amplitude is of the order of a $1 / 10^{\text {th }}$ the amplitude of the $\mathrm{P}$ wave. Also the polarity of atrial repolarisation is opposite to depolarisation indicating that repolarisation 'propagation' follows a similar pathway to depolarisation and PAF does not appear to alter this pattern. 

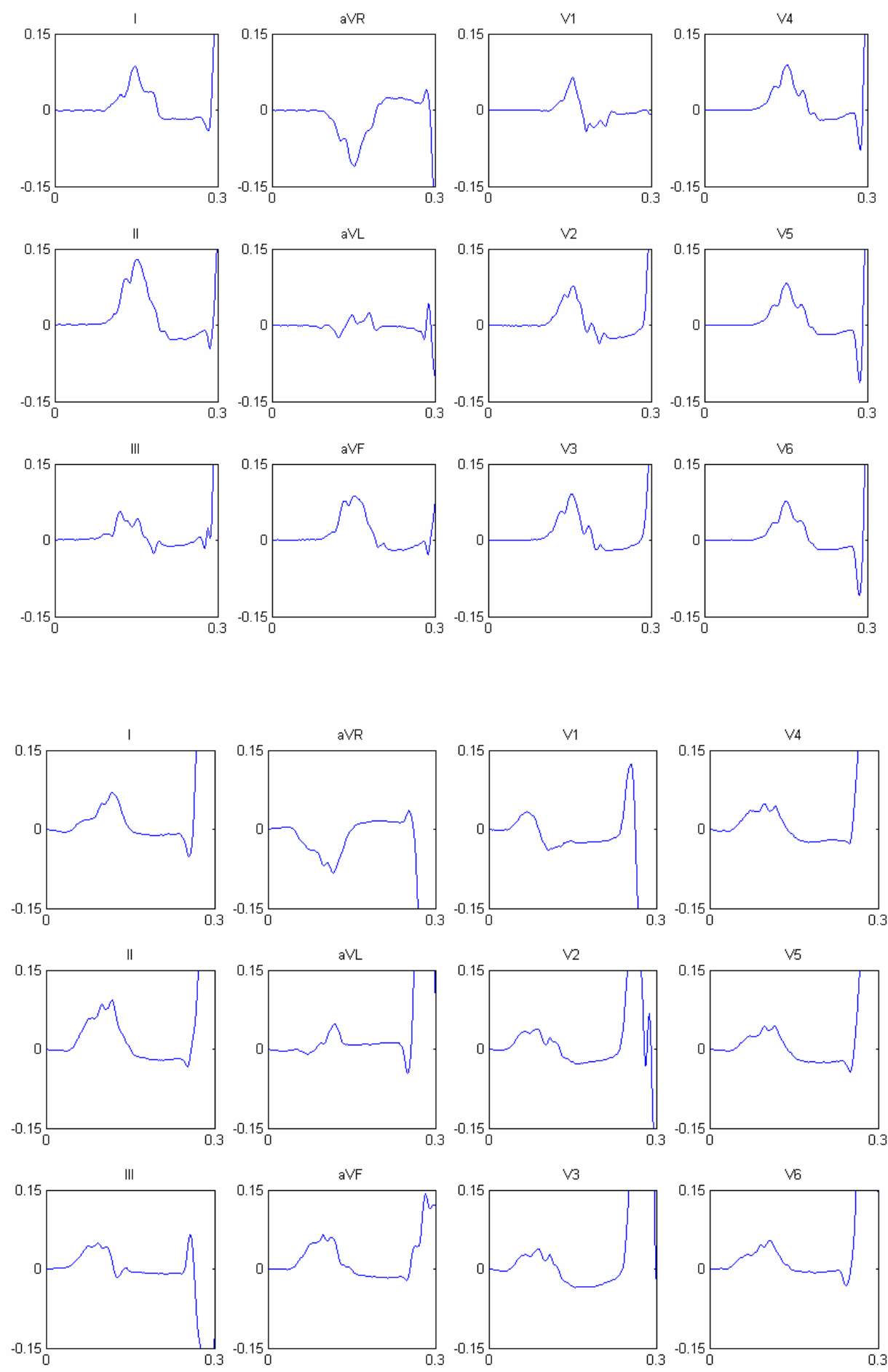

Figure 3. Average atrial beat in each lead of a healthy subject (top panel) and PAF patient (bottom panel). Amplitude shown in $\mathrm{mV}$, time scale is $0.3 \mathrm{~s}$. 

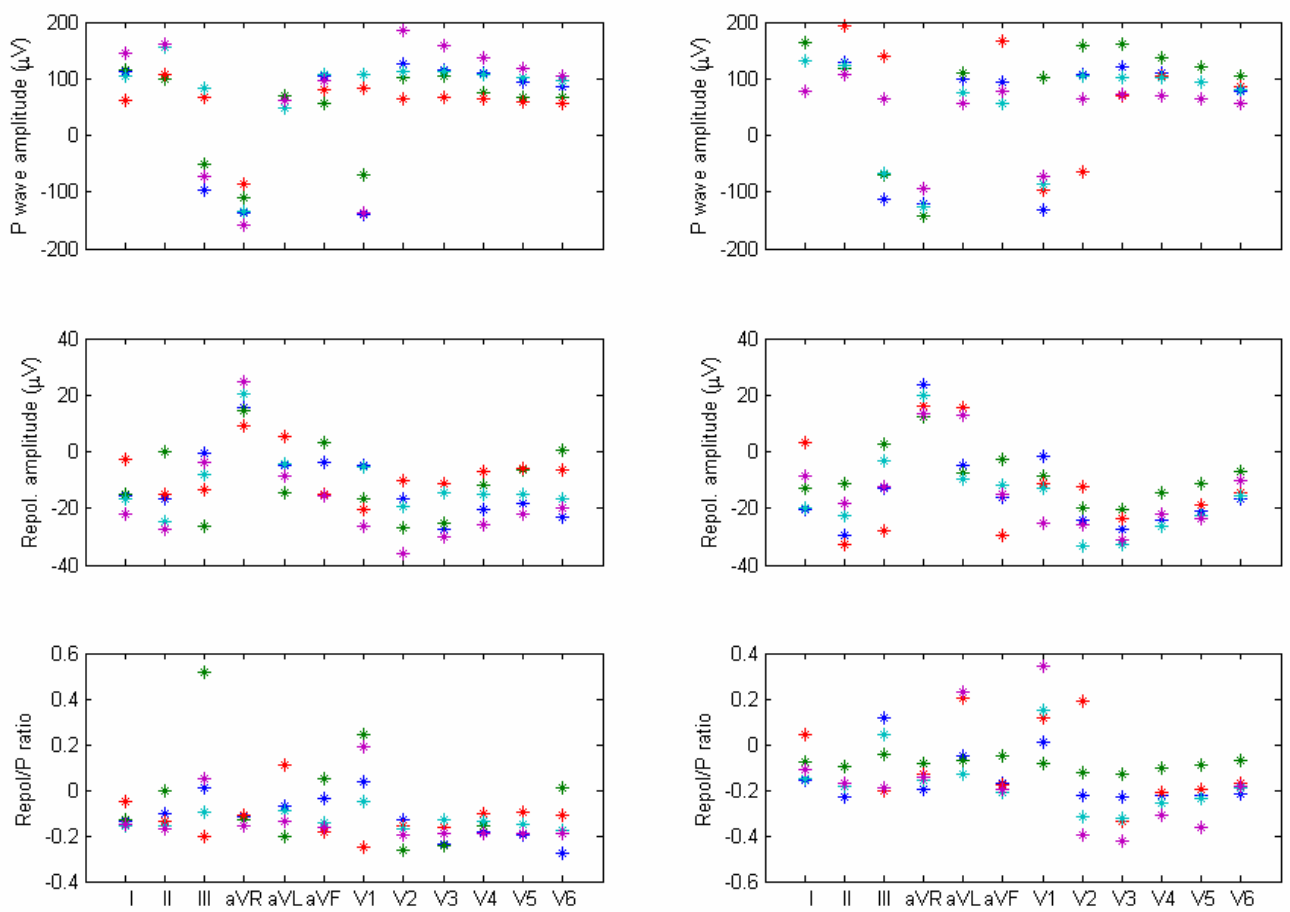

Figure 4. P wave and atrial repolarisation (Repol) amplitude measurements and ratio for healthy (left column) and PAF (right column) groups.

\section{Acknowledgements}

PL is supported by an EPSRC Advanced Research Fellowship.

\section{References}

[1] Broady DA, Arzbaecher RC, Woolsey DM, Sato T. The normal electrocardiogram: Morphology and quantitative variability in bipolar extremity leads. Am. Heart J.1967;74:4-12.

[2] Li Z, Hertervig E, Yuan S, Yang Y, Lin Z, Bertil Olsson S. Dispersion of atrial repolarisation in patients with paroxysmal atrial fibrillation. Europace 2001;3:285-91.
Address for correspondence

Philip Langley

Medical Physics Dept.

Freeman Hospital

Newcastle upon Tyne

NE7 7DN

U.K.

philip.langley@ncl.ac.uk 